\title{
Impacto producido por la técnica seoi-otoshi. Relación con años de práctica y grado en judo
}

\author{
Carlos MONTERO CARRETERO*1,2 \& José Luis LÓPEZ ELVIRA ${ }^{1}$ \\ ${ }^{1}$ Centro de Investigación del Deporte. Universidad Miguel Hernández de Elche (España) \\ ${ }^{2}$ Escuela De Frutos, Ozone Sports Club, San Juan de Alicante (España)
}

Recepción: 10/10/2013; Aceptación: 01/04/2014; Publicación: 04/04/2014.

ARTÍCULO ORIGINAL

\section{Resumen}

La técnica seoi-otoshi (popularmente, seoi-nage de rodillas) es frecuentemente utilizada por los judokas en sus entrenamientos. Se debate sobre si los más jóvenes deberían realizarla por la repercusión que causarían los impactos sobre sus estructuras en formación. Nuestro objetivo fue relacionar el impacto contra el suelo al ejecutar la técnica seoiotoshi con los años de experiencia y el grado. Participaron 33 judokas, divididos en tres grupos según los años de práctica. Dos plataformas de fuerza bajo el tatami registraron las fuerzas de impacto durante la realización de cinco repeticiones consecutivas, se extrajo el promedio de los picos y su máximo. Los resultados muestran fuerzas de hasta 10 veces el peso corporal, posiblemente de riesgo en jóvenes, especialmente si son repetidas en el tiempo. Además, se observa una tendencia a la baja de los impactos producidos a medida que el judoka aumenta los años de práctica (relación potencial; $\mathrm{R}^{2}=0.41, \mathrm{p}<0.000$ para el pico de fuerza), $\mathrm{y}$ las fuerzas obtenidas en el grupo de expertos es significativamente menor que en los otros ( $\mathrm{p}<0.001)$. Sin embargo, con el grado se aprecia una relación cuadrática, que hace que aumente al principio y disminuya en los niveles superiores $\left(\mathrm{R}^{2}=0.45\right.$, $\mathrm{p}<0.000$ para el pico de fuerza). La falta de concordancia entre años de práctica y grado indica que los criterios de promoción no parecen adecuados en cuanto a garantizar la correcta ejecución desde el punto de vista preventivo, especialmente en los grados más bajos, que inevitablemente se corresponderán con practicantes más jóvenes, en los que el aparato locomotor se encuentra en desarrollo.

Palabras clave: Judo; biomecánica; entrenamiento; riesgo de lesión; maduración; deportes de combate.

\section{Impact produced by seoi-otoshi technique.} Relation to years in practice and judo rank

\begin{abstract}
Judokas commonly train the seoi-otoshi technique (aka, drop-knee seoi-nage). A controversy exists about the convenience of its use by the younger judokas due to the risk of high loads produced by the impacts on their growing structures. The aim of the present paper was to measure the impacts against the tatami when executing the knee seoi-otoshi technique and its relationship with the years of practice and the degree or level (color of the belt). Thirty-three judokas from different years and degree volunteered to participate. Two force plates covered by standard tatami, registered the ground reaction forces while five consecutive repetitions were executed. We measured the mean and maximum peaks of force relative to their own body weight (BW). The results show peaks of more than $10 \mathrm{BW}$, which can be considered a potential risk of injury in the younger judokas, especially when repeated in time. In addition, a tendency to decrease the impact as the years of practice increase is observed (potential
\end{abstract}

Impacto produzido pela técnica seoi-otoshi. Relação com os anos de prática e graduação no judo

\section{Resumo}

A técnica seoi-otoshi (popularmente conhecida por seoi-nage de joelhos) é frequentemente utilizada pelos judocas nos seus treinos. Existe um debate sobre se os mais jovens deveriam realizá-la pela repercussão e os impactos causados sobre a sua estrutura óssea em formação. 0 nosso objetivo foi o de relacionar o impacto com o solo ao efetuar a técnica seoi-otoshi com os anos de experiência e a graduação. Participaram neste estudo 33 judocas, divididos em três grupos, segundo os anos de prática. Duas plataformas de força debaixo o tatami registraram as forças de impacto durante a realização de cinco repetições consecutivas, extraindo-se a média dos picos e o seu máximo. Os resultados mostram forças de impactos até 10 vezes superior o peso corporal, as quais sejam possivelmente perigosas pelos mais jovens, especialmente se são repetidas com o tempo. Por outro lado, observa-se uma tendência baixa dos impactos produzidos à medida que o judoca aumenta os anos de prática (relação potencial; $\mathrm{R} 2=0.41, \mathrm{p}<0.000$ para o pico de força) e as forças obtidas para o grupo de especialistas é significativamente menor do que nos outros $(\mathrm{p}<0.001)$. Com

*E-mail: cmontero@umh.es. Dirección: Centro de Investigación del Deporte de la Universidad Miguel Hernández de Elche. Avenida de la Universidad, s/n. 03202. Elche (España). 
function; $R^{2}=0.41, p<0.000$ in the force peak), and the force in the expert group has been significantly lower than in the other groups $(\mathrm{p}<0.001)$. On the other hand, the degree (belt color) shows a quadratic relationship ( $\mathrm{R}^{2}=0.45, \mathrm{p}<0.000$ in the force peak). The lack of agreement between the years of practice and the degree shows that the promotion criteria does not appear to be a valid one from a preventive viewpoint, especially in the lower degrees which would correspond to younger practitioners whose locomotor structures are still not fully developed.

Keywords: Judo; biomechanics; training; injury risk; maturation; combat sports. a graduação, verifica-se uma relação quadrática, que faz com que aumente ao princípio e diminua com os níveis superiores $\left(\mathrm{R}^{2}=0.45, \mathrm{p}<0.000\right.$ para o pico de força). A falta de concordância entre os anos de prática e a graduação indica que os critérios de promoção não parecem adequados, quanto a garantir a correta execução do ponto de vista preventivo, especialmente nas graduações mais baixas, que inevitavelmente correspondem com os praticantes mais jovens, quando a estrutura do aparato locomotor se encontra ainda em desenvolvimento.

Palavras-chave: Judo; biomecânica; risco de lesão; treino; maturidade; desportos de combate.

\section{1.- Introducción}

El judo es un deporte de combate, que se practica sobre una superficie acolchada (tatami) donde los deportistas comienzan de pie y tratan de ejecutar técnicas con el fin de proyectar al contrincante sobre su espalda (nage-waza). En el caso de que la proyección no sea perfecta, la lucha se continúa en suelo (ne-waza), con el propósito de alcanzar la victoria a través de técnicas de inmovilización, luxación o estrangulación (Pocecco, Gatterer, Ruedl, \& Burtscher, 2012). El número de técnicas propias de esta disciplina y las diferentes características de las mismas es muy elevado (Yiannakis, 2011), por lo que múltiples autores han intentado clasificarlas en función de diferentes criterios (Castarlenas \& Calmet, 1999). Dentro de las técnicas de proyección, existen algunas en las que el judoka se apoya de rodillas sobre el tatami con el fin de provocar el desequilibrio y posterior volteo del adversario. Estas técnicas han suscitado bastante controversia entre los profesionales del judo, por la posibilidad de provocar lesiones en los practicantes que las realizan, hecho por el cual han sido incluidas y excluidas por los diferentes reglamentos a lo largo del tiempo, en algunos lugares de España, en categorías de edades tempranas (alevines, menos de 13 años).

Las características de las propias técnicas, el nivel de dominio del deportista, las reacciones que se producen por oposición directa de los adversarios, el nivel de entrenamiento y la magnitud de los impactos que se generan sobre el organismo, son algunos factores determinantes en el riesgo de lesión. En judo, el grado de dominio se refleja por el color del cinturón, de modo que en general los judokas más noveles lucen el cinturón de color blanco, y van adquiriendo cinturones de color amarillo, naranja, verde, azul y marrón, previamente a obtener el cinturón negro o primer Dan. El tiempo que transcurre entre uno y otro grado no está determinado exactamente por los estamentos federativos, y los programas para su obtención varían en función del lugar donde se realice el proceso de formación. No obstante, generalmente pasan varios años desde que el judoka se inicia en la práctica hasta que opta al cinturón negro, con lo que el tiempo de práctica hasta la obtención del mencionado grado suele sobrepasar los 10 años. Ericcson, Krampe y Tesch-Römer (1993) establecen que ese es el mínimo de años que un deportista necesita realizar práctica deliberada, para alcanzar el grado de experto, en línea con la clásica "regla de los 10 años" de Simon y Chase (1973).

Los exámenes de paso de grado a cinturón negro realizados por parte de la Real Federación Española de Judo y Deportes Asociados (RFEJYDA) se fundamentan en la demostración de gran número de técnicas y fundamentos que dan a entender la adquisición de habilidades y conocimientos variados sobre los componentes técnicos del judo. Dentro de las técnicas requeridas está la conocida como seoi-nage (figura 1a). Esta técnica es una de las más usadas por los judokas en competición desde edades tempranas (Carratalá, García, \& Fernández, 2009), aunque a menudo es empleada la modalidad de esa técnica de rodillas, cuyo nombre real es seoi-otoshi aunque sea conocida entre el colectivo de judokas como seoi-nage de rodillas (figura 1b). Esta modalidad implica dejarse caer de rodillas y supone un impacto más agresivo con el tatami. Si la técnica no es ejecutada correctamente, se puede transmitir a estructuras poco preparadas para absorber impactos, como las propias rodillas, caderas y columna lumbar. 
A nivel general, se ha constatado que el $12.2 \%$ de las lesiones en competiciones de judo afectan a la rodilla (Frey et al., 2004), siendo acciones traumáticas responsables de ruptura del ligamento cruzado anterior en un 5.6\% de los casos (Kobayashi et al., 2010). De hecho, se considera a las técnicas basadas en seoi-nage como de riesgo para las rodillas del ejecutante en todas las categorías, tanto en hombres como en mujeres (Pocecco et al., 2013). Con todo, los judokas de diferentes niveles parecen conceder gran importancia a la competición (Cepeda, Montero, García, León, \& Iglesias, 2011), por lo que entrenan diariamente estas técnicas para preparar los campeonatos.

Figura 1. a) Técnica seoi-nage de pie; b) Técnica seoi-otoshi.

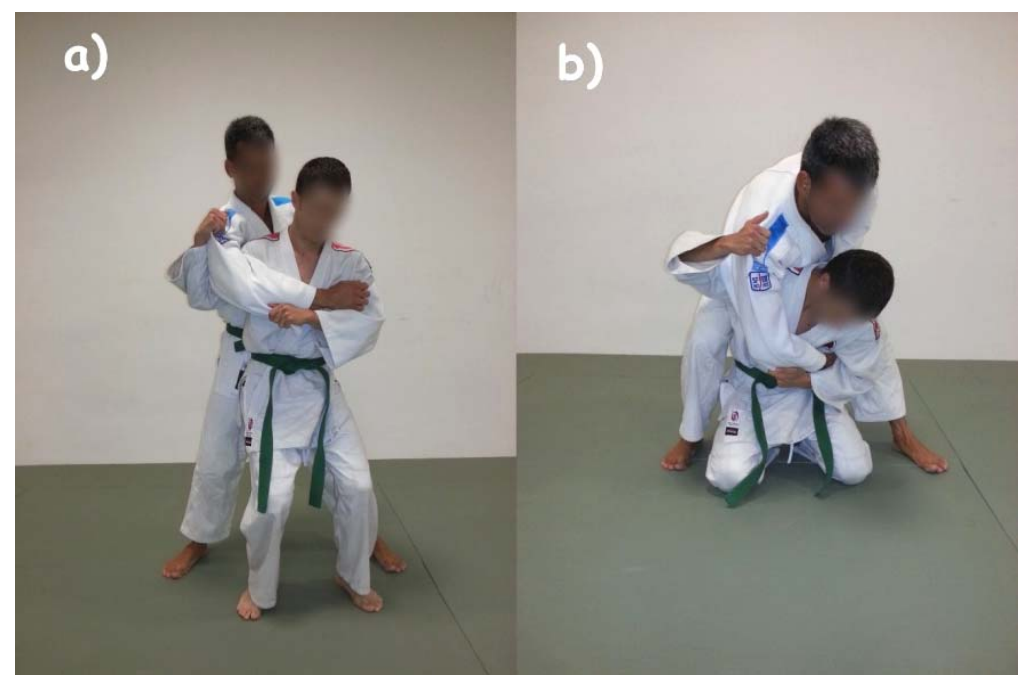

Dentro de los diferentes métodos de entrenamiento de las técnicas de proyección tradicionalmente empleados en judo y recogidos en los manuales clásicos de esta disciplina (Kano, 1986), destaca el uso del conocido como uchi-komi, que consiste en repetir las fases de desequilibrio y preparación (kuzushi y tsukuri) de los gestos técnicos con el fin de automatizarlos en primera instancia, e incrementar las capacidades condicionales tales como fuerza, velocidad o resistencia en etapas posteriores. Otros métodos de entrenamiento a través de los cuales se realizan las diferentes técnicas llegando a proyectar a uke es el nage-komi, el kakari-geiko, o el randori. Todos estos métodos son empleados por los judokas en los entrenamientos diarios pudiendo ser el número de repeticiones de un gesto técnico muy elevado en función del nivel del deportista y el grado de exigencia del entrenador. Por este motivo, todos los judokas que incluyen la técnica seoi-otoshi en sus repertorios técnicos acumularían un gran volumen de impactos sobre sus rodillas.

En otros ámbitos se ha estudiado la relación entre los picos de fuerza en el impacto y la aparición de fracturas por estrés. Concretamente en la carrera de larga distancia en mujeres, se encontró que los impactos eran mayores y más rápidos en corredoras que habían sufrido fracturas por estrés en las tibias que en un grupo control (Milner, Ferber, Pollard, Hamill, \& Davis, 2006). Esto indica una asociación entre impactos repetidos, aunque sean de intensidad moderada, y la aparición de fracturas por estrés. En judo la intensidad de los impactos puede ser mayor que en la carrera, y ciertos métodos de entrenamiento implican acciones de impacto repetidas en el tiempo. Al igual que sucede en otras acciones deportivas, la técnica juega un papel primordial en la capacidad de amortiguación (Weerdesteyn, Groen, van Swigchem, \& Duysens, 2008).

Se ha estudiado con frecuencia el valor máximo de fuerza vertical en el impacto con el suelo producido en otras técnicas deportivas. Como referencia se presentan algunos ejemplos de actividades representativas: en la carrera de velocidad alcanza 3 veces el propio peso corporal (PC) del sujeto (Novacheck, 1998); en la caída de un remate en voleibol 4.25 PC (Bisseling, Hof, Bredeweg, Zwerver, \& Mulder, 2008); en la caída de un rebote en baloncesto 7.1 PC (Valiant \& Cavanagh, 1985); en la caída de un doble salto mortal en gimnasia 14.4 PC (Panzer Wood, Bates, \& Mason, 1988) y 15.2 BW en un triple salto (Perttunen, Kyrolainen, Komi, \& Heinonen, 2000). Sin 
embargo, no se han encontrado estudios que aporten información sobre el volumen ni la intensidad de los impactos producidos en las distintas técnicas de judo, o si los judokas que han empleado este tipo de técnicas han sufrido un mayor número de lesiones o de mayor gravedad que aquellos que no las hayan practicado o lo hayan hecho en menor medida. En referencia al estudio del impacto que suponen algunos movimientos en judo, se ha comprobado el beneficio del uso de técnicas de caída propias del judo a la vida cotidiana, bajo la premisa de que una correcta técnica de caída podría reducir los impactos y así el riesgo de sufrir una lesión. Concretamente se ha estudiado el impacto producido sobre la cadera ante una caída lateral desde una posición de rodillas cayendo en bloque o realizando la caída lateral de judo (yoko-ukemi) rodando (Groen, Weerdesteyn, \& Duysens, 2007). En ese estudio se encontró que la caída lateral en bloque producía fuerzas de unos 2.7 PC, que recaían principalmente sobre la cadera. Tras un periodo de entrenamiento en la correcta técnica de judo, las mismas personas conseguían reducir el impacto a apenas 2 PC, con el consiguiente beneficio, especialmente en personas en riesgo de fracturas por caídas, como son las personas de la tercera edad.

Teniendo en cuenta lo anteriormente expuesto, parece justificada la preocupación por parte de los profesionales del judo en torno a si puede resultar lesivo el empleo de la técnica de proyección seoi-otoshi en jóvenes judokas en periodos de formación ósea. Aunque no existe consenso sobre en qué edades es mayor el riesgo de sufrir una lesión (Harmer, 2010), se ha constatado una mayor ratio de judokas lesionados entre los 16 y los 20 años (Frey et al., 2004), cuando los judokas son muy combativos pero aún inmaduros técnica y tácticamente (Pocecco et al., 2013).

Por todo ello, y ante la ausencia de estudios que hayan abordado el tema, el objetivo del presente trabajo fue analizar el impacto que produce la técnica seoi-otoshi en judokas y su relación con los años de experiencia y el grado de dominio, identificado por el cinturón.

\section{2.- Método}

\section{1.-Participantes}

Participaron un total de 33 judocas ( 29 hombres y 4 mujeres) de distintas procedencias, en un intento de representar distintos niveles en cuanto a años de práctica, edad y grado (tabla 1). Todos los participantes estaban familiarizados con la técnica seoi-otoshi. Previamente firmaron un consentimiento informado de acuerdo con la declaración de Helsinki de 1975 y 2000.

Tabla 1. Características descriptivas de la muestra de cada grupo y del conjunto de todos los participantes.

\begin{tabular}{lccccc}
\hline & Edad (años) & Talla (m) & Masa (kg) & Cinturón* & Años de práctica \\
\hline Inexpertos $(\mathrm{n}=11)$ & $21.6 \pm 4.7$ & $1.72 \pm 0.11$ & $67.3 \pm 9.6$ & $5.2 \pm 1.8$ & $1.2 \pm 0.7$ \\
Iniciados $(\mathrm{n}=11)$ & $11.7 \pm 1.3$ & $1.49 \pm 0.11$ & $48.1 \pm 12.3$ & $5.6 \pm 2.4$ & $5.6 \pm 1.1$ \\
Expertos $(\mathrm{n}=11)$ & $20.1 \pm 9.0$ & $1.67 \pm 0.06$ & $65.2 \pm 8.5$ & $9.8 \pm 1.5$ & $14.9 \pm 8.1$ \\
TODOS $(\mathrm{n}=33)$ & $17.8 \pm 9.0$ & $1.63 \pm 0.14$ & $60.2 \pm 13.2$ & $6.9 \pm 2.8$ & $7.2 \pm 7.4$ \\
\hline
\end{tabular}

*Para el cinturón se aplicó un valor ordinal, correspondiendo el 1 al blanco y el 11 al negro.

\section{2.- Protocolo}

Para medir las fuerzas de reacción del suelo, se emplearon dos plataformas de fuerzas Kistler 9287CA (90x60 cm, Kistler, Winterthur, Suiza) colocadas en paralelo formando una superficie de registro de 121 x $90 \mathrm{~cm}$. La frecuencia de registro empleada fue de $1000 \mathrm{~Hz}$.

Sobre las plataformas se colocó una colchoneta de tatami estándar de $4 \mathrm{~cm}$ de grueso y una densidad de $250 \mathrm{~kg} / \mathrm{m}^{3}$. Como la colchoneta tenía un tamaño superior al del área abarcada por las plataformas, parte de la fuerza vertical ejercida por los judokas podría dispersarse por los extremos apoyados en el suelo del contorno. Para reducir al mínimo este efecto, la superficie de las plataformas se elevó $1 \mathrm{~cm}$ respecto al suelo circundante y se ajustaron dos lados de la colchoneta a los de las plataformas, haciendo coincidir una esquina. Además, se pidió a los judokas que intentaran ajustarse al lado de la colchoneta que no mantenía contacto con el suelo externo. En un 
estudio piloto en el que se pesó en distintas posiciones un objeto de masa conocida, se comprobó que esta estrategia permitía medir la fuerza vertical correctamente en el espacio necesario para ejecutar la técnica requerida.

Los participantes actuaron por parejas, alternándose los papeles de tori y uke (ejecutante y oponente respectivamente). Tras realizar un calentamiento estándar se les pesaba por separado. Para realizar las técnicas, ambos se colocaban en la posición natural de judo (shizen-hon-tai) con agarre (kumi-kata) fundamental de manga y solapa, cada uno sobre una plataforma con la indicación de que al inicio permanecieran dentro de ella. Tori repetía 5 veces consecutivas la técnica seoi-otoshi, en forma de uchi-komi, mientras uke mantenía la posición sin mover los pies de la plataforma. Se realizó este número de repeticiones para tener en cuenta la variabilidad inherente a los seres humanos (Preatoni et al., 2013) pero sin entrar en zona de fatiga, especialmente en los más jóvenes.

\section{3.- Análisis de datos}

Se analizó la componente vertical de la fuerza de reacción del suelo durante la ejecución. En la figura 2 puede verse un ejemplo de las fuerzas registradas en una sola repetición y su correspondencia con las posiciones de los judokas durante de la ejecución.

Figura 2. Registro de fuerzas verticales realizado por ambas plataformas. 1) Inicio de la técnica cuando el pie derecho despega del suelo; 2) Despegue del pie izquierdo que libera la plataforma de tori; 3 ) Inicio de la fase de vuelo; 4) Instante del máximo impacto.
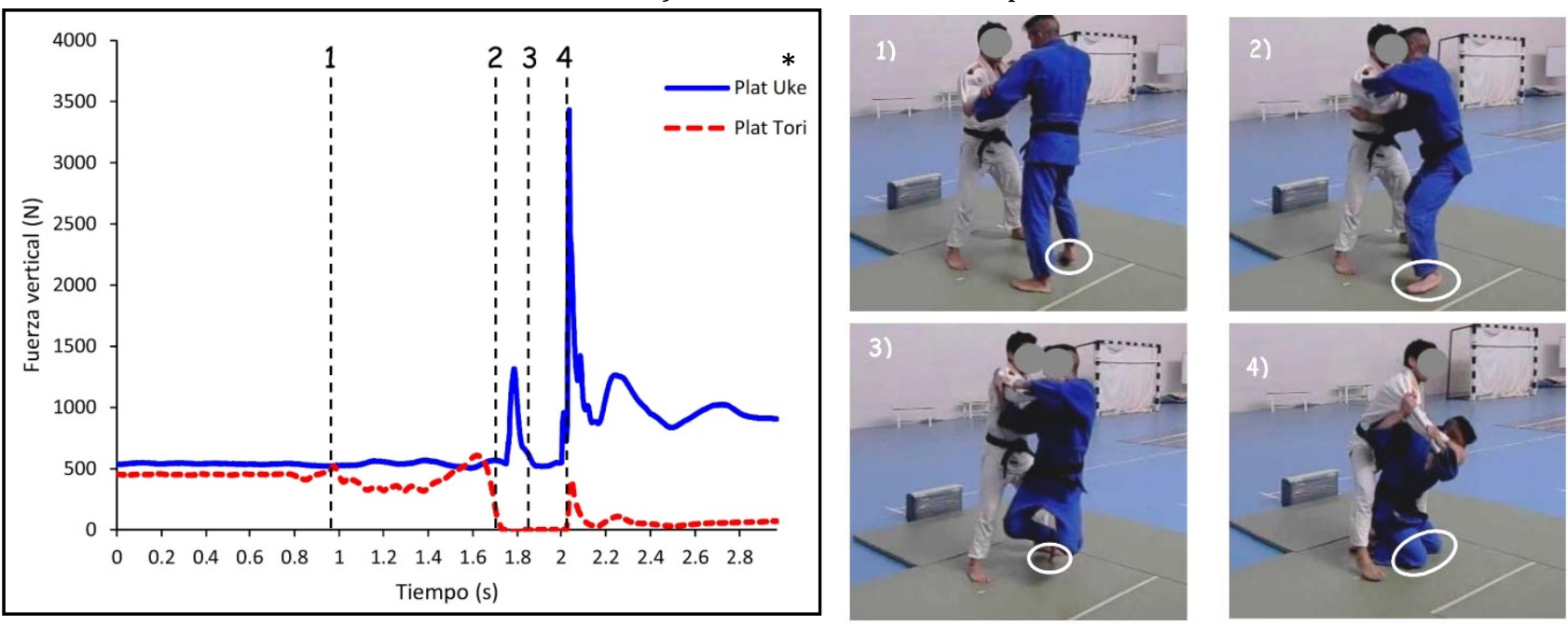

* PlatTori representa la plataforma sobre la que se encuentra tori al inicio de la técnica, mientras que PlatUke representa la plataforma sobre la que se encuentra uke. Una vez iniciado el movimiento, el peso de tori se distribuye entre ambas plataformas.

Se sumó la fuerza obtenida en las dos plataformas y se le restó el peso de $u k e$, para así tener constancia de las fuerzas producidas por la acción de tori. A pesar que de uke tenía instrucciones de permanecer quieto, es posible que en algunos casos se haya movido, contaminando de algún modo el registro de fuerza producida exclusivamente por tori. Sin embargo, consideramos más adecuado mantener las condiciones del experimento lo más ecológicas posible, ya que si uke se mueve y se apoya en tori incrementando las fuerzas de reacción, sigue siendo fuerza que tienen que soportar las estructuras de tori, por lo que también supondrían un impacto potencialmente lesivo que entra dentro de nuestro objeto de estudio.

Se normalizaron las fuerzas respecto al propio peso corporal (PC) de cada ejecutante (cuando actuaba como tori) para anular el efecto del peso en las diferencias inter-sujeto (Jansen et al., 2012). Sobre esa señal, se buscaron los valores máximos de las cinco repeticiones, correspondientes al instante del impacto (figura 3). 
Figura 3. Ejemplo de registro de fuerzas en un sujeto. A la suma de las dos plataformas se le ha restado el peso de $u k e$. Se ha marcado con círculos los picos correspondientes a los cinco impactos.

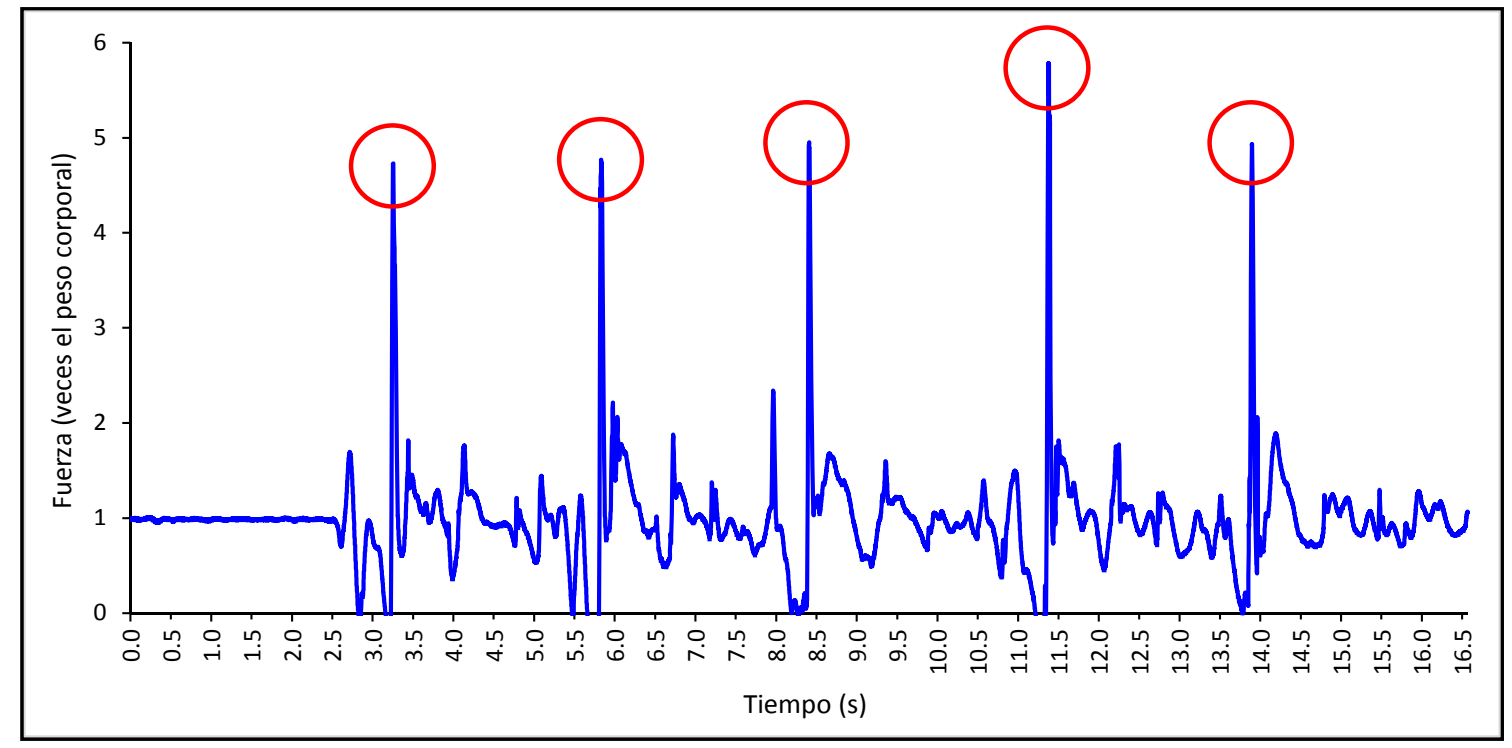

Como variables dependientes se analizaron el promedio de los picos de las cinco ejecuciones (representativo de la técnica habitual), y el pico máximo de las cinco repeticiones (relacionado con el máximo riesgo de lesión). Como variables independientes se tomaron, por un lado, el grado, a partir del cinturón según una escala ordinal de 1 a 11, y por otro lado los años de práctica de judo.

\section{4.- Análisis estadístico}

Con el fin de establecer la relación entre las variables dependientes e independientes, se realizó un análisis de regresión. Las curvas de regresión contrastadas fueron: lineal, cuadrática, logarítmica, potencial y exponencial. Se calculó el valor $\mathrm{R}^{2}$ como indicativo del porcentaje de varianza en una variable explicado por la otra. Se eligió la curva que mejor se ajustaba en cada caso a partir del valor de $\mathrm{R}^{2}$ más alto.

También se realizó una clasificación de la muestra en tres grupos dividiendo por terciles según los años de práctica (11 sujetos por grupo), resultando las siguientes agrupaciones: Inexpertos, con menos de 3 años de práctica; Iniciados, con entre 3 y 8 años de práctica; Expertos, con 8 o más de práctica. Se realizó la prueba de normalidad de Shapiro-Wilk, mostrando todas las variables una distribución normal. Se calculó un ANOVA de un factor para comparar los tres grupos entre sí. En el caso de encontrar diferencias se realizó una comparación por pares con el test post hoc de Bonferroni. El análisis estadístico se realizó con el programa SPSS versión 20.0 (IBM SPSS Inc., Chicago, IL).

\section{3.- Resultados}

En la figura 4 se representan los resultados relativos a la comparación entre los impactos y los años de práctica por un lado, y el grado a partir del cinturón por otro, tanto para el valor máximo de fuerza como para el promedio de las cinco repeticiones.

Las curvas de regresión se calcularon a partir del mejor ajuste según el estadístico $\mathrm{R}^{2}$. La relación con los años de práctica se obtuvo con una regresión potencial, que indica que el pico de fuerza obtenido se explica a partir de los años de práctica en un $37.94 \%$ y en un $41.54 \%$ (fuerza promedio y pico de fuerza respectivamente), $\mathrm{p}<0.000$. Por otra parte, la relación con el grado se obtuvo con una regresión cuadrática, que indica que el pico de fuerza obtenido se explica a partir de los años de práctica en un $37.37 \%$ y en un $45.74 \%$ (fuerza promedio y pico de fuerza respectivamente), $\mathrm{p}<0.000$. 
Figura 4. Relación entre el pico de impacto promedio (a y c) y máximo (b y d) con los años de práctica (a y b) y con el grado (c y d). La curva de regresión con los años de práctica se realiza con una regresión potencial, mientras que la del grado se representa con una regresión cuadrática (en todos los casos p<0.000).

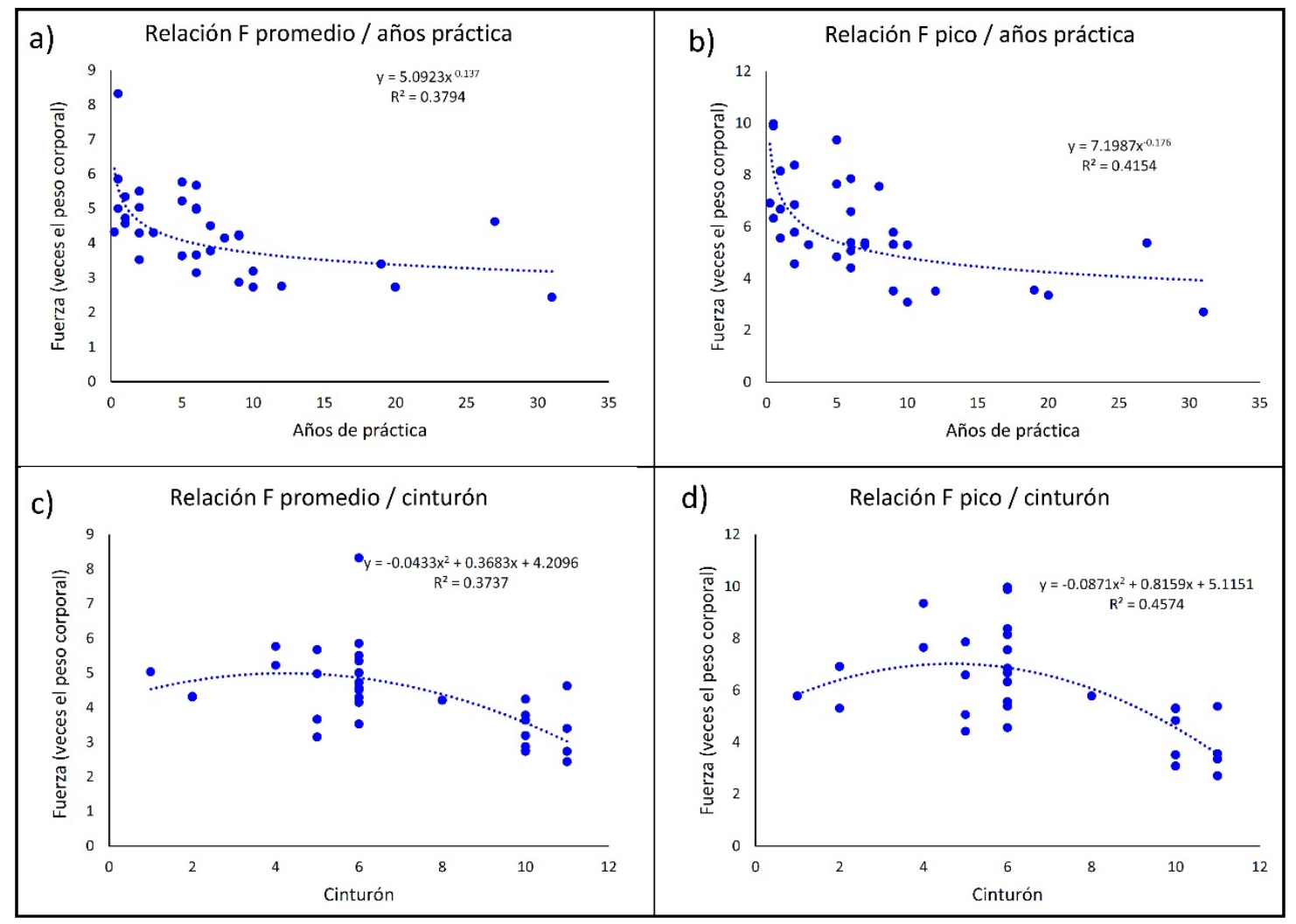

En la figura 5 se presentan los resultados de la comparación de medias dividiendo a los sujetos en tres grupos según una distribución en terciles a partir de los años de práctica (en cada grupo $\mathrm{n}=11$ ). Los resultados indican que se encontraron diferencias significativas en el grupo de expertos respecto a los otros dos.

Figura 5. Comparación de medias entre los tres grupos en función de los años de práctica. Inexpertos: menos de 3 años; Iniciados: 3 o más y menos de 8; Expertos: más de 8 años. *p<0.001.

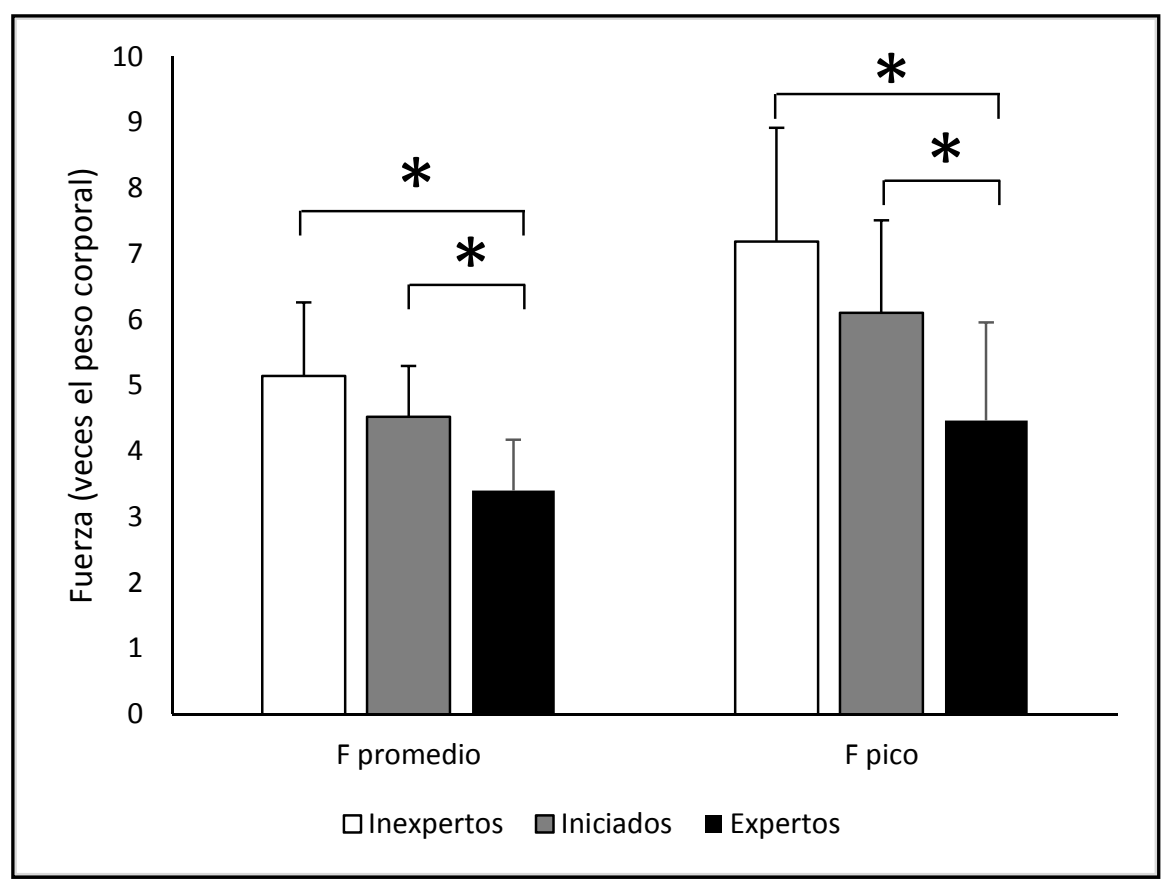




\section{4.- Discusión y conclusiones}

El principal objetivo del estudio fue analizar el impacto que produce la técnica seoi-otoshi en judokas y su relación con los años de experiencia y el grado de dominio, con el fin de abordar la controvertida temática de lo adecuado de su uso en judokas de edades tempranas. Los resultados muestran que los años de práctica explican en un $37.94 \%$ y en un $41.54 \%$ la fuerza promedio y pico de fuerza respectivamente, mostrándose una tendencia a la baja de los impactos producidos a medida que el judoka cuenta con más años de práctica. Este resultado se confirma por las diferencias significativas encontradas entre el grupo de expertos y los otros dos grupos $(\mathrm{p}<0.001)$. No obstante, los resultados no permiten afirmar que exista una relación directa, de modo que a más años de práctica menor impacto al realizar el seoi-otoshi. Tal como se puede observar en las figuras $4 \mathrm{a}$ y $\mathrm{b}$, en algunos casos aquellos que han practicado durante más años, producen impactos, promedio y pico, mayores que los que llevan menos tiempo realizando judo. Esto puede deberse a muchas causas, entre otras a que el número de repeticiones de esta técnica concreta a lo largo de su vida deportiva puede variar sustancialmente de unos a otros, aspecto no tenido en cuenta en este trabajo. Tampoco hemos diferenciado entre las metodologías de aprendizaje de dicha técnica, ya que en algunas escuelas podría enseñarse teniendo muy en cuenta el modo de aterrizar sobre el tatami, mientras en otras esto no sería contemplado como un aspecto relevante.

Con la intención de encontrar variables que puedan explicar el mayor o menor impacto producido, analizamos este en relación con el grado de dominio del judoka. Así, al considerar el grado que marca el color del cinturón, cabría esperar que a mayor grado más dominio de la técnica, y por tanto menor impacto debería producirse en la caída. Sin embargo, aunque los resultados muestran una tendencia a descender, no lo hacen de forma lineal sino parabólica (figura 4c y d), encontrando los valores mayores no en los grados menores sino en los intermedios. Este resultado podría generar controversia si entendemos que un mayor grado de domino debería reportar un menor impacto sobre el organismo del deportista. En cualquier caso, no hemos analizado aspectos como la velocidad en la ejecución, la efectividad o fuerza aplicada sobre el adversario, que suelen ser menores en deportistas noveles por falta de destreza y confianza. Sería interesante estudiar si existe relación entre la eficacia de la ejecución y el impacto producido, esto es, si se debe producir un impacto determinado para que la técnica sea efectiva, o por el contrario puede ser igualmente efectiva produciendo impactos menores, lo cual sería recomendable para la salud de los judokas. Parece razonable que el objetivo de la técnica consista en ser capaz de transmitir al oponente la fuerza generada para derribarlo, y no de transmitirla al suelo a través del impacto de los propios segmentos corporales.

Por otro lado, entendiendo que el color del cinturón determina el grado de dominio del judoka, cabría preguntarse si realmente éste mide la cantidad o calidad de los conocimientos y habilidades adquiridas por el judoka que se presenta a un examen de paso de grado. Hay que recordar que el programa de acceso a cinturón negro, se fundamenta en el conocimiento de gran número de técnicas, pero no distingue entre un cinturón blanco y un primer Dan por la calidad con la que se ejecuta una de ellas.

Finalmente, cabe destacar que algunos de los participantes de la muestra obtuvieron valores de hasta 10 PC, y que son muchos los que pasaron de 5 PC. Estos datos confirman las sospechas de los profesionales del judo a cerca de los riesgos que puede suponer la realización de esta técnica en determinados deportistas, y justifican los cambios que se han producido a lo largo del tiempo en los reglamentos. Los impactos registrados se encuentran al nivel de los de la caída de un remate en voleibol (4.25 PC, Bisseling et al., 2008) o de la caída tras un rebote en baloncesto (7.1 PC, Valiant y Cavanagh, 1985). Podemos afirmar que el impacto producido por los judokas al realizar la técnica seoi-otoshi es considerable, más si entendemos que en las tareas anteriormente descritas, los deportistas producen ese impacto al contactar con el suelo sobre las plantas de los pies calzados, mientras que en el caso de la técnica seoi-otoshi estos se producen en primera instancia sobre las rodillas.

A pesar de sus limitaciones, este estudio aporta información relevante para los deportistas y entrenadores de judo, y trata de concienciarlos acerca de la importancia de prestar atención a los impactos producidos al realizar las técnicas de rodillas, por las posibles implicaciones que podría tener en la salud de los judokas. 
Como limitaciones del estudio, la muestra empleada es escasa sobre todo en algunos rangos de edad, especialmente en los más expertos. Al agrupar a los sujetos, la edad del grupo de iniciados resulta ser menor que la de los otros dos, hecho que puede haber influido de alguna forma en los resultados. Sin embargo esto responde a nuestra intención de mantener una amplia representatividad en la muestra. Por otra parte, las fuerzas recibidas por tori pueden haber estado contaminadas por los movimientos de uke al recibir la técnica, a pesar de que los ukes fueron instruidos para que evitaran moverse. En cualquier caso, consideramos que la importancia sobre las variables medidas es despreciable. Es posible que los resultados en cuanto a picos de fuerza en el impacto estén relacionados con la forma de enseñar la técnica. En estudios futuros sería interesante analizar las diferencias entre distintos centros de enseñanza del judo, así como comprobar si se pueden reducir los impactos a través de la mejora de la técnica tras un entrenamiento adecuado. Además, futuros estudios deberían analizar la relación entre la efectividad de la técnica y el impacto producido al ejecutarla.

\section{5.- Aplicaciones}

Los resultados del estudio permiten llamar la atención a los entrenadores sobre los altos niveles de impacto que se producen al realizar la técnica seoi-otoshi. Especialmente en practicantes en crecimiento, debería prestarse atención a la enseñanza y entrenamiento de la técnica de caída, haciendo hincapié en la aplicación de los criterios de prevención de lesiones, como la amortiguación. Este esfuerzo en la enseñanza de la técnica debe realizarse puesto que se ha visto que el cambio de grado en sí no garantiza la mejora en la reducción de impactos, al menos en las primeras etapas.

\section{Nota}

Una versión previa de este trabajo se presentó en el I Congreso Internacional de Judo De FrutosUMH, Alicante, 27-30 de junio de 2013.

\section{Referencias}

Bisseling, R. W., Hof, A. L., Bredeweg, S. W., Zwerver, J. y Mulder, T. (2008). Are the take-off and landing phase dynamics of the volleyball spike jump related to patellar tendinopathy? British Journal of Sports Medicine, 42(6), 483-489.

Carratalá, V., García, J.M., \& Fernández, L. (2009). Análisis de las acciones técnicas de los judokas cadetes participantes en el campeonato de España. Revista Internacional de Ciencias del Deporte, 15(5), 64-80.

Castarlenas, J.L. \& Calmet, M. (1999). Aspectos conceptuales del judo: clasificación y enseñanza de sus contenidos. En M. Villamón (Ed). Introducción al judo (pp. 263-310). Barcelona: Hispano Europea.

Cepeda, M., Montero, C., García, L., León, D., \& Iglesias, D. (2011). La percepción de judocas de distinto nivel de pericia respecto a las actividades de entrenamiento específicas que contribuyen al rendimiento. Archivos de Medicina del Deporte, 18, 257-264.

Groen, B. E., Weerdesteyn, V., \& Duysens, J. (2007). Martial arts fall techniques decrease the impact forces at the hip during sideways falling. Journal of Biomechanics, 40(2), 458-462.

Ericsson, K. A., Krampe, R. T. , \& Tesch-Römer, C. (1993). The role of deliberate practice in the acquisition of expert performance. Psychological Review, 100, 363-406.

Frey, A., Rousseau, D., Vesselle, B., Hervouet Des Forges, Y., \& Egoumenides, M. (2004). Neuf saisons de surveillance médicale de compétitions de judo: Une analyse nationale de la traumatologie du judo en compétition. Journal de Traumatologie du Sport, 21(2), 100-109.

Janssen, I, Sheppard, J. M., Dingley, A. A., Chapman, D. W., \& Spratford, W. (2012). Lower extremity kinematics and kinetics when landing from unloaded and loaded jumps. Journal of Applied Biomechanics, 28, 687-693.

Kano, J. (1986). Kodokan judo. Tokyo: Kodansha International Ltd. 
Kobayashi, H., Kanamura, T., Koshida, S., Miyashita, K., Okado, T., Shimizu, T., \& Yokoe, K. (2010). Mechanisms of the anterior cruciate ligament injury in sports activities: a twenty-year clinical research of 1,700 athletes. Journal of sports Science and Medicine, 9(4), 669-675.

Milner, C.E., Ferber, R., Pollard, C.D., Hamill, J., \& Davis, I.S. (2006). Biomechanical factors associated with tibial stress fracture in female runners. Medicine and Science in Sports and Exercise, $38(2), 323-328$.

Novacheck, T.F. (1998). The biomechanics of running. Gait \&Posture, 7(1), 77-95.

Panzer, V.P., Wood, G.A., Bates, B.T., \& Mason, B.R. (1988). Lower extremity loads in landings of elite gymnasts. En G. de Groot, A. Hollander, P. Huijing, \& G. Van Ingen Schenay (Eds.), Biomechanics XI-B, (pp. 694-700). Amsterdam: Free University Press.

Perttunen, J. O., Kyrolainen, H., Komi, P. V., \& Heinonen, A. (2000). Biomechanical loading in the triple jump. Journal of Sports Science, 18(5), 363-370.

Pocecco, E., Gatterer, H., Ruedl, G., \& Burtscher, M. (2012). Specific exercise testing in judo athletes. Archives of Budo, 8(3), 133-139.

Pocecco, E., Ruedl, G., Stankovic, N., Sterkowicz, S., Del Vecchio, F. B., Gutierrez-Garcia, C., Rousseau, R., Wolf, M., Kopp, M., Miarka, B., Menz, V., Krusmann, P., Calmet, M., Malliaropoulos, N., \& Burtscher, M. (2013). Injuries in judo: a systematic literature review including suggestions for prevention. British Journal of Sports Medicine, 47(18), 1139-1143.

Preatoni, E., Hamill, J., Harrison, A. J., Hayes, K., Van Emmerik, R.E.A., Wilson, C., \& Rodano, R. (2013). Movement variability and skills monitoring in sports. Sports Biomechanics, 12(2), 6992.

Simon, H. A. \& Chase, W. G. (1973). Skill in chess. American Scientist, 61, 394-403.

Valiant, G. A. \& Cavanagh, P. R. (1985). A study of landing from a jump: implications for the design of a basketball shoe. En D. A. Winter, R. W. Norman, R. P. Wells \& K. C. Hayes (Eds.), Biomechanics $I X-B$ (pp. 117-122). Champaign, Illinois: Human Kinetics.

Weerdesteyn, V., Groen, B.E., van Swigchem, R., \& Duysens, J. (2008). Martial arts fall techniques reduce hip impact forces in naive subjects after a brief period of training. Journal of Electromyography and Kinesiology, 18(2), 235-242.

Yiannakis, L. (2011). Una taxonomía de los principios utilizados en las técnicas de proyección de judo. Revista de Artes Marciales Asiáticas, 6(2), 69-84.

\section{Authors' biographical data}

Carlos Montero Carretero (España), es Doctor en Psicología y especialista en motivación deportiva. Licenciado en Ciencias de la Actividad Física y el Deporte, Entrenador Nacional de Judo y 5o Dan, actualmente es profesor de Deportes de Lucha en la Universidad Miguel Hernández de Elche y Director-Entrenador del De Frutos Club de Judo de Alicante. Como competidor del Equipo Nacional de Judo fue Bicampeón de España Senior, Bronce en el Campeonato del Mundo Universitario, y Bronce en Campeonato de Europa por Equipos. E-mail: cmontero@umh.es.

José Luis López Elvira (España), es Licenciado en Educación Física por la Universidad de León y especializado en Biomecánica, desarrolló su tesis doctoral en la misma universidad, centrada en el estudio desde el punto de vista biomecánico de una modalidad de lucha local, la lucha leonesa. En la actualidad es profesor titular en la Universidad Miguel Hernández de Elche. E-mail: jose.lopeze@umh.es. 